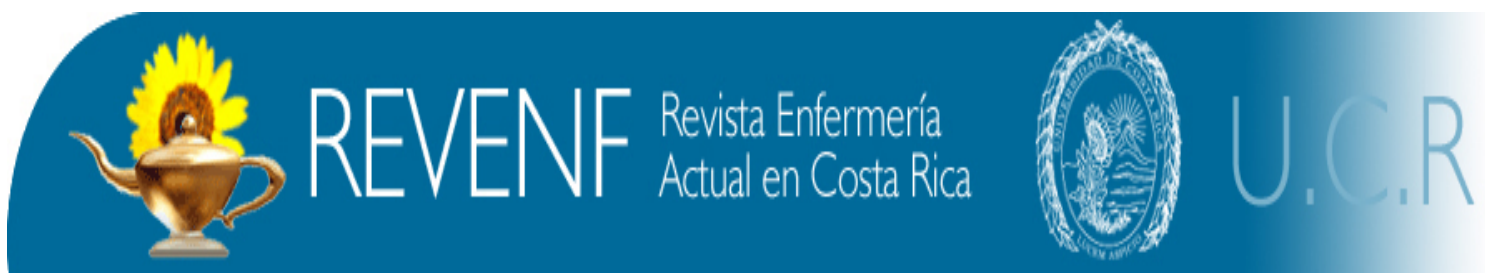

REVISTA SEMESTRAL, No. 10 Año 5

ISSN1409 - 4568

\title{
ESTILOS DE VIDA SALUDABLE Y NO SALUDABLE QUE TIENEN LOS Y LAS TRABAJADORAS DEL NUCLEO DE PROCESOS ARTESANALES DEL INSTITUTO NACIONAL DE APRENDIZAJE ${ }^{1}$
}

Licda. Irene Álvarez Quesada ${ }^{2}$ Licda. Angela Cristina Lara Escalante

Licda. Monserrat Navarro Mora

\begin{abstract}
RESUMEN
El presente artículo tiene como objetivo presentar los resultados obtenidos de un estudio sobre la promoción de estilos de vida saludable en la población que labora en el Núcleo de Procesos Artesanales del Instituto Nacional de Aprendizaje (INA). Surge a partir de la evolución del concepto salud-enfermedad, del cambio en el perfil epidemiológico de Costa Rica, así como de la necesidad de disminuir el riesgo de enfermedad y promover la salud; esto sumado a las características propias del lugar donde se desarrolló la investigación. Se implementó una metodología cuantitativa, de tipo descriptivo-transversal. Se aplicó los principios que enmarca la operacionalización múltiple, ya que se emplearon técnicas de recolección de información cualitativas y cuantitativas como son la observación no participante, historial de enfermería y cuestionarios estructurados autoadministrados.

Los problemas de la población en estudio encontrados en el diagnóstico están relacionados con las siguientes temáticas: estrés, proceso salud enfermedad y los estilos de vida saludable, tabaquismo, alcoholismo, ejercicio, alimentación balanceada, autoestima y recreación.
\end{abstract}

Palabras claves: Proceso salud-enfermedad, estilo de vida, andragogía, educación en salud, Enfermería.

\footnotetext{
${ }^{1}$ Fecha de recepción: Enero - 2006

${ }^{2}$ Licenciadas en Enfermería. San José, Costa Rica.
}

Fecha de aceptación: Marzo - 2006 


\begin{abstract}
The present article must as objective present/display the results obtained of a study on the promotion of styles of healthful life in the population that toils in the Nucleus of Artisan Processes of the Instituto Nacional de Aprendizaje(INA). Health-disease arises from the evolution of the concept, the change in the profile epidemiologist of Costa Rica, as well as of the necessity to diminish the disease risk and of promoting the health; this added to the own characteristics of the place where the investigation was developed. A quantitative methodology was implemented, of descriptive-cross-sectional type. It was applied the principles that the multiple operacionalization frames, since qualitative and quantitative techniques of information harvesting were used as they are the non-participant, historial observation of nursing and structured questionnaires. The problems of the population in study found in the diagnosis are related to following the thematic ones: stress, process health - disease and the styles of healthful life, tobacco, alcoholism, exercise, balanced feeding, self-esteem and recreation.
\end{abstract}

KEY WORDS: Process health-disease, style of life, education of adults, education in health, Nursing. 


\section{INTRODUCCIÓN}

Esta artículo hace referencia a los resultados obtenidos luego del diagnóstico de necesidades educativas sobre los estilos de vida saludable y el posterior desarrollo de un programa de enfermería para promoverlos. Se desarrolló en el Núcleo de Procesos Artesanales del Instituto Nacional de Aprendizaje, sede Hatillo, con la participación de las personas que laboran de forma continua en dicho Núcleo.

El estudio surgió debido a la observación que realizaron las investigadoras en donde desarrollaron prácticas clínicas. A partir de ese momento se cuestiona sobre la continua evolución del ser humano; ya que éste al igual que el medio que lo rodea y las diversas situaciones en las que se encuentra inmerso, constituyen verdaderos procesos de cambio y dinamismo que van progresando con el tiempo; como se observa en la realidad nacional, primeramente con respecto al concepto salud, donde ésta deja de ser exclusivamente ausencia de enfermedad y se convierte en un proceso de construcción social por medio del cual cada individuo se convierte en el actor protagónico de su propia salud; en segundo lugar, la transición del perfil epidemiológico de Costa Rica, según el cual las principales causas de muerte ya no son de tipo infeccioso sino por enfermedades crónicas; por último, el ambiente en que se desarrollan tanto profesional como personalmente los costarricenses.

La nueva conceptualización del proceso salud-enfermedad, considera cuatro diferentes dimensiones las cuales son: dimensión biológica (factores biológicos, genéticos, inmunes y la enfermedad en sí), dimensión de conciencia y conducta (hábitos, creencias, costumbres, ideas, relaciones interpersonales, ética, valores del individuo y formas de conceptualización social de la salud y la enfermedad), dimensión ecológica (entorno) y dimensión económica (solvencia económica del individuo). 
Al respecto Martínez y Sánchez afirman que:

"El proceso salud-enfermedad es así, el resultado de la relación dinámica entre los procesos biológicos, ecológicos, culturales, económicos y sociales que se dan en la sociedad. Sobre la base de esta nueva conceptualización, la salud ya no será responsabilidad única del sector salud $y$ en consecuencia, deben involucrarse diferentes actores y sectores sociales” (Martínez, R. y Sánchez, R. 1999: p. 99)

Por lo tanto, toma relevancia y asume su razón de ser la promoción de la salud, la cual la define el Ministerio de Salud de Costa Rica como:

"Una estrategia fundamental para actuar sobre los determinantes de la salud y los estilos de vida individuales y colectivos, otorgándole valor a la salud, la que requiere de esfuerzos sectoriales e intersectoriales, en conjunto con la comunidad, para preservarla, mantenerla y mejorarla. Comprende las siguientes áreas de intervención: políticas públicas saludables, participación social, fomento de estilos de vida saludables, seguridad alimentaria y nutricional” (Ministerio de Salud 2003: p. 51).

Los estilos de vida consideran tanto el contexto en el cual los individuos y grupos sociales se desarrollan cotidianamente como las experiencias que estos viven; además, de la relación recíproca entre estos dos aspectos. Entonces por el concepto de estilos de vida se entiende:

"Las formas de conducirse tales como los hábitos o los comportamientos sostenidos, de los individuos y los grupos sociales y que son resultado de la interacción de factores de tipo material (vivienda, alimentación, vestido, servicios de saneamiento básico y tecnología), social (organización a la que se pertenece) e ideológico (ideas, valores y creencias), los cuales proporcionan las condiciones que determinan un estado de salud y la prolongación de la vida” (Villalobos, LB 1989, p. 60).

Dentro de los factores que incluye el concepto de estilos de vida están: la alimentación, la obesidad, el ejercicio, la recreación, el estrés, el descanso, el tabaquismo, el alcoholismo y las enfermedades crónicas. 
Este estudio está dirigido a las personas adultas, por lo que fue pertinente abordar el tema de la andragogía, según Adam (1987), etiológicamente la palabra andragogía es un término derivado de la raíz “andro” que significa “hombre adulto” y “gogía” que significa dirigir. Entonces andragogía es: “Toda aquella actividad conducida intencional y profesionalmente, que aspira cambiar a las personas adultas; el cambio que se produce o se dirige es considerado como algo deseable y que se percibe como algo bueno o como superación” (Adam, F. 1987, p. 2). Es por ello, que para la promoción de la salud y la prevención de enfermedades en poblaciones adultas se emplea la andragogía, la cual considera el bagaje de conocimientos y experiencias de cada individuo.

El papel de enfermería en la educación para la salud, se centra principalmente, en brindarle a la persona las herramientas, técnicas y conocimientos para que por sí mismo y misma, pueda discernir entre aquellos aspectos que favorecen o no su calidad de vida y así poder realizar los ajustes necesarios, en su respectivo estilo de vida. Por lo tanto, "El rol de la enfermera en la promoción de la salud consiste en identificar los factores que aumentan el riesgo del adulto joven de contraer problemas de salud, y en educarle y apoyarle para que reduzca las conductas que ponen en riesgo su salud” (Potter y Perry 2003: p. 234).

Por consiguiente, la enfermería como profesión es única porque es la que transmite las respuestas sobre los problemas de salud a los individuos y los familiares. Además, las y los enfermeros aprenden tanto de la experiencia y las actitudes como de las teorías de enfermería, encontrando la manera de aplicarlas a la práctica, ya que estas pueden constituir la base del planeamiento del cuidado. 


\section{OBJETIVOS}

\section{OBJETIVO GENERAL}

Caracterización de los estilos de vida saludable y no saludable en los y las trabajadoras del Núcleo de Procesos Artesanales del Instituto Nacional de Aprendizaje, sede Hatillo.

\section{OBJETIVOS ESPECIFICOS}

1. Describir el ambiente laboral de los y las trabajadoras del Núcleo de Procesos Artesanales del Instituto Nacional de Aprendizaje.

2. Identificar los estilos de vida del personal del Núcleo de Procesos Artesanales del Instituto Nacional de Aprendizaje.

\section{MATERIALES Y MÉTODOS}

En esta investigación se realizó un análisis de los estilos de vida de la población en estudio. Se trabajo desde un enfoque cuantitativo, de tipo descriptivo-transversal; el cual describe a la población a partir de datos estadísticos; no obstante, como parte de la caracterización de una población es esencial la utilización de técnicas cualitativas que permitan acercarse a la realidad. Por esto, se utilizó la operacionalización múltiple.

Los datos se obtuvieron a partir del historial de enfermería, cuestionarios autoadministrados y observación no participante cuyos datos se registraron por medio de una hoja de cotejo.

En este estudio se trabajó con el total de la población de los y las trabajadoras del Núcleo de Procesos Artesanales del Instituto Nacional de Aprendizaje. Dicha población, según datos brindados por la doctora Alfaro, directora del Núcleo, está conformada por veinte personas. 


\section{RESULTADOS Y DISCUSIÓN}

El análisis de los resultados se realizó según el orden de los objetivos planteados en este estudio.

En relación con el ambiente laboral, se evidencia que el 70\% de los y las trabajadoras se sienten motivados al iniciar la jornada laboral. Cabe destacar que la motivación es un sentimiento relacionado con la satisfacción en el desempeño de sus labores y además con las buenas relaciones interpersonales que mantienen.

Por otro lado, el 65\% de los y las participantes describieron su lugar de trabajo como bonito, lo cual se pudo verificar con la observación realizada por las investigadoras. El 70\% de los y las trabajadoras refieren estar satisfechas con el ambiente laboral y el 60\% están motivadas. Por otra parte, en lo que concierne al espacio físico del lugar de trabajo, el 75\% de las y los sujetos de estudio se sienten cómodos. A pesar de que al 70\% les gustaría cambiar algún aspecto de éste, dentro de los cuáles están, la distribución del espacio y la ventilación. Al respecto, durante la observación no participante, se evidenció que esta última es inadecuada debido a la poca funcionalidad de las ventanas, ya que las mismas permanecen cerradas o no se pueden abrir. Al respecto Aguilar Herrera (1992: s.p.) afirma que la ventilación implica tanto cantidad como la calidad del aire suministrado ya que desde el punto de vista de la salud, el problema de la ventilación debe necesariamente comprender la calidad de aire. Aspectos que se observan en el ambiente laboral del Núcleo ya que no sólo las entradas de aire son insuficientes sino que además la calidad del mismo no es adecuado debido a la ubicación del Núcleo, por factores como el humo emitido por 
los automóviles que transitan por los alrededores, residuos de madera del taller de maderas y emisión de gases provenientes de los talleres de metal, pintura y estética.

En relación con la distribución del espacio, este estudio pudo evidenciar que algunas oficinas tienen una sola persona, mientras que en otras se observó hacinamiento, ya que tienen cuatro o seis personas; dicha distribución se debe al tipo de trabajo que desempeñan. Los y las trabajadoras afirmaron en un $90 \%$ que cuentan con espacio para realizar sus actividades laborales. Además, el 65\% de ellos y ellas refieren contar con adecuada iluminación y con buen aseo.

Con respecto a la iluminación, se observaron ventanas y fuentes de luz artificial en cada uno de los lugares de trabajo; según Ivancevich, J. \& Matteson, M. (1989: p.120) una buena iluminación se percibe como alegre y estimulante y, por tanto relajante ya que tiene menor probabilidad de actuar como estresor.

Por otro lado, un 30\% perciben ruido en su lugar de trabajo; sin embargo, las investigadoras detectaron abundante ruido proveniente de diversas fuentes. En relación con esto, se afirma, que el ruido aparentemente opera con menos intensidad como estresor en las situaciones en las que es excesivo pero esperado, que en aquellas en las que o no se espera o es por lo menos impredecible (Ivancevich, J. \& Matteson, M. 1989: p.125).

Otro aspecto relevante para este estudio comprendió el análisis de las medidas de seguridad con las que cuenta el Núcleo, en relación a esto el 55\% de las personas respondió que el edificio no tiene medidas de seguridad. Se determinó la ausencia de salidas de emergencia, de extintores y rótulos de emergencia. Además, el Núcleo está estructurado únicamente con dos accesos, de los cuales uno de ellos se encuentra con llave, por motivos de seguridad contra el hampa. 
Para el segundo objetivo se obtuvieron los siguientes resultados: en relación con la dimensión económica, según los ingresos que reciben mensualmente la población en estudio, se determinó que se encuentra en un rango de 100 mil a más de 300 mil colones; donde el 65\% recibe un salario superior a los 250 mil colones. Además, el 75\% de los y las trabajadoras no asumen por sí solas los gastos económicos del Núcleo familiar, es decir contribuyen a los gastos del hogar entre dos y cuatro personas.

Por otro lado, el 85\% de la población afirma que el ingreso es relativamente suficiente para suplir las necesidades básicas de sus familias; además, algunas de ellas consideran que de los ingresos depende mucho el tener una buena o mala calidad de vida. En ese sentido, manifestaron también en un 95\% que tienen algún tipo de deuda financiera.

En lo referente a la dimensión ecológica, el 30\% de los participantes residen en áreas rurales y manifestaron que éstas pueden favorecer un estilo de vida saludable, al ofrecer mayor cantidad de áreas verdes para el esparcimiento, la relajación y la recreación; además, de ser un lugar con menores fuentes de contaminación, sónica, del aire, agua, entre otros. Con respecto a lo anterior se afirma que “...un ambiente exterior de aire puro, agua limpia, vegetación hermosa, ruido mínimo y poca densidad de población, supondría un avance importante hacia la relajación” (Melgosa, J. 1997: p.58). Asimismo, el 100\% de la población en estudio cuentan en su comunidad con servicios de agua potable, recolección de desechos sólidos y zonas verdes; en un 70\% manifestaron que su comunidad es un lugar seguro, lo cual beneficia directamente la calidad de vida de cada uno de ellos y ellas.

De acuerdo con la dimensión biológica, el $80 \%$ de los y las trabajadoras tienen como antecedente patológico familiar la hipertensión, el 60\% el alcoholismo y en un 50\% 
obesidad. Dichos antecedentes predisponen a los y las participantes a padecer en un futuro estas enfermedades dependiendo del estilo de vida que tengan.

Por otra parte, el 75\% de ellos y ellas desayunan y más del 50\% de los y las trabajadoras del Núcleo realizan diariamente los cinco tiempos de comida (desayuno, merienda, almuerzo, merienda y cena); este aspecto se considera importante dentro de un estilo de vida saludable, ya que al realizar cinco tiempos se fraccionan más las comidas y se disminuye la cantidad ingerida; lo cual contribuye a mantener un metabolismo activo y así, prevenir enfermedades nutricionales como la obesidad. De acuerdo con lo anterior Castro, L. y otros, afirma que: "Los hábitos alimentarios condicionan, positiva o negativamente el estado de salud de un individuo o de una población. De esta manera, si se trata de hábitos inadecuados, se está contribuyendo a la prevalencia de alguna enfermedad asociada con la nutrición (por ejemplo: obesidad, enfermedades cardiovasculares, etc.)” (Castro, L.; Peralta, T.; Quesada, C. \& Sibaja, A. 1997: p.6).

De acuerdo con las características del sueño, el 80\% de la población refiere una sensación de descanso al despertar y un $75 \%$ afirma que su sueño es profundo; el $80 \%$ duerme diariamente más de seis horas. Estos hábitos relacionados con el patrón del sueño, benefician la salud pues le permite a la persona descansar y recuperar las energías invertidas a lo largo del día. Según lo afirma Gerin, S. (1998) los seres humanos duermen alrededor de un tercio de su vida ya que la media de horas que se duerme por noche es de ocho horas, lo cual representa más de 121 días al año dedicados a las horas sueño; ello pone de manifiesto la importancia que se le debe otorgar al sueño y, sobre todo, la influencia que éste ejerce sobre el resto de nuestras actividades y nuestra salud, ya que el sueño constituye un período esencial de recuperación física y psíquica. Sin embargo, un $80 \%$ de los y las 
participantes dedican solamente una hora o menos para descansar diariamente; la actividad más frecuente con la que descansan es viendo televisión, para un 60\%.

Asimismo, el 55\% de los y las trabajadoras acostumbran salir a pasear los fines de semana. Con lo cual, el tiempo que dedican a descansar es reducido para realmente recuperarse de las actividades diarias realizadas, como así lo mencionaron los y las participantes. De acuerdo con Melgosa, J. (1997), el descanso cotidiano debe ser de calidad con el fin de poder recuperarse del desgaste físico y psíquico; además el descanso semanal es de suma importancia para prevenir el estrés; por ejemplo, hay algunas personas que aprovechan el sábado y el domingo para continuar y ampliar la misma labor que han estado realizando durante la semana laboral. Esto representa un gran peligro, ya que puede acabar en un estrés acentuado. También, por medio de actividades vacacionales se rompe con la rutina de las labores cotidianas, con lo cual se restaura la energía.

Por otro lado, con respecto a la actividad física el $65 \%$ de los sujetos de este estudio acostumbran caminar como acción implícita en el diario quehacer y un 50\% realiza las actividades domésticas, las cuales son consideradas actividades físicas. Dichas actividades se derivan de su entorno inmediato es decir, el trabajo y el hogar. Al respecto, la Organización Panamericana de la Salud (2002) define la actividad física como un término amplio que incluye todo tipo de movimientos y abarca actividades que varían en intensidad desde subir las escaleras habitualmente, bailar y caminar, hasta correr, montar bicicleta y practicar deportes. Además, del total de la población en estudio, el 55\% de los y las trabajadoras expresaron no realizar ningún tipo de ejercicio físico; aspecto que podría estar repercutiendo en su estilo de vida; ya que según Melgosa (1997), la persona que realiza ejercicio físico habitual cuenta con mayores ventajas a la hora de reposar, ya que el corazón 
bombea más lentamente durante el reposo regenerándose mejor. Se concilia más fácilmente el sueño cuando se practica ejercicio físico con regularidad, y es una de las estrategias de afrontamiento más eficaces contra el estrés.

Según la dimensión de conciencia y conducta, el 40\% de los y las trabajadoras del Núcleo realizan actividades recreativas una ó dos veces por semana y el 30\% realiza estas actividades al menos dos ó tres veces por semana. De lo anterior, Salazar Salas, C. (1997) refiere que nuestra sociedad está caracterizada por un acelerado estilo de vida, donde algunos individuos trabajan constantemente sin otorgarse un espacio para descansar.

En lo referente al tema del estrés laboral, para el 30\% de la población en estudio una de las situaciones que les genera estrés es el tiempo / plazo con el que cuentan para entregar o realizar un trabajo. El 15\% afirma que le genera estrés la cantidad de trabajo que se les asigna. Al respecto se señala lo siguiente:

\footnotetext{
"El contenido del trabajo, la estructura organizacional así como la personalidad y las capacidades de los trabajadores, pueden todos ellos conducir a un estrés laboral... El estrés experimentado, a menudo no se debe a un único estresor sino a la interacción entre múltiples estresores” (O’Brien, G.1998: p. 72).
}

De los sujetos de estudio quienes sufren estrés, el 55\% lo manifiesta al padecer dolores musculares y el 25\% al presentar cambios en el carácter. Potter, B. (1991) recalca que el agotamiento y estrés laboral comienza con ligeras señales como: sentimientos de frustración, crisis emocionales, retraimientos, problemas de salud, desempeño deficiente y consumo de drogas o alcohol. Si estos síntomas se desatienden pueden progresar hasta que la persona sienta aversión por ir a trabajar. El 25\% utiliza como estrategia de afrontamiento ante el estrés, hablar, ya sea con sus superiores, compañeros o familia; un $15 \%$ realiza ejercicios, otro $15 \%$ practica la autoterapia y va a sesiones de masajes un $15 \%$. Para 
minimizar los efectos del estrés, existe una amplia gama de estrategias; Quirós, R. (1994) menciona entre estas: hacer ejercicio, mejorar la alimentación, meditar, práctica de masajes, baños calientes, pedir apoyo y reír.

Otro aspecto interesante, fue lo referente al autoconcepto, en donde el 20\% de las y los sujetos de estudio se describen a sí mismos como luchadores y el 20\% como personas tranquilas. Además, según la descripción de la imagen corporal, el 30\% de ellos y ellas se aceptan como son y el 25\% les gustan como se ven al mirarse al espejo. Igualmente, el 95\% se sienten orgullosos de ellos y ellas mismas. También el 25\% afirma que sus compañeros los y las perciben como personas amigables, el 15\% como buena gente y otro 15\% creen que los y las perciben como sujetos agradables. Los aspectos anteriormente mencionados son importantes en la vida de todo ser humano; ya que como afirma Potter y Perry (2003), lo que el individuo piensa y sienta sobre sí mismo afecta la forma en que se cuida física y emocionalmente y la forma en que es capaz de cuidar a los demás. También definen el autoconcepto, como el conocimiento de un individuo sobre sí mismo, una mezcla de pensamientos conscientes e inconscientes, sentimientos, aptitudes y percepciones. Está constituido por la identidad, la imagen corporal y la autoestima.

Dentro de los hábitos no saludables el 40\% de la población en estudio afirmó consumir bebidas alcohólicas. De esas personas, el 37.5\% las ingieren únicamente en eventos sociales y un 25\% cada fin de semana; con respecto a la cantidad que consumen el 50\% ingieren de tres a cuatro "tragos” / “cervezas”; refieren como algunas razones de este hábito las siguientes: que les gusta, sabe rico, por el ambiente, para celebrar, para liberar la tensión, por estrés, diversión, por la compañía, por motivos sociales, como parte de la conversación, por indicación médica, entre otras. 
Al respecto el Instituto de Alcoholismo y Fármacodependencia menciona que:

"Los peligros relacionados con el uso del alcohol disminuyen notablemente con el establecimiento de hábitos de bebida. La mayor parte de las personas que beben se amoldan a lo dispuesto por los grupos a los cuales pertenecen. A pesar de lo anterior, los riesgos siempre existen. El alcohol es considerado regular y preponderantemente como una bebida social; sin embargo, no se puede asegurar que no se llegue a mirarlo y usarlo como una droga. El alcoholismo y la ebriedad son prueba de lo anterior” (IAFA 1983, p. 13).

El 50\% de quienes ingieren bebidas alcohólicas negaron tener alguna repercusión en sus vidas como consecuencia de este hábito. Por el contrario, un 25\% comunicaron que la ingesta de estas sustancias tenía repercusiones de tipo personal, para el $12.5 \%$ las repercusiones eran de tipo familiar y para el otro $12.5 \%$ lo son de tipo laboral. En lo referente al fumado, únicamente el $25 \%$ de los y las trabajadoras manifestó que fuma tabaco, de los y las cuales, el $40 \%$ lo hace diariamente y otro $40 \%$ cada fin de semana. De quienes fuman un $60 \%$ refirió consumir de 10 cigarrillos a un paquete diario.

Además, los y las fumadoras comunicaron practicar este hábito por: cuestiones sociales, para liberar tensión, por ansiedad, por estrés y para acompañar al alcohol. Al respecto López Jiménez, H. (1993) afirma que el hábito del fumado es un ciclo que oscila entre tensión, fumado, alivio y relajación. Desafortunadamente aquí no termina el ciclo ya que a través de la cronicidad de la adicción, el fumado y la tensión aumentarán en tanto que el alivio y la relajación decrecen. Igualmente, de quienes consumen tabaco el 80\% negó tener repercusiones.

Otro aspecto relevante para este estudio comprendió el análisis del concepto de salud, en donde se logró determinar que el mayor porcentaje, es decir, un 75\% lo definen 
como: sentirse bien física, emocional, espiritual, laboral y familiarmente. Igualmente, se abordó la conceptualización de la enfermedad, donde el 20\% de la población la definió como: algo malo que da pavor; un 15\% como un desequilibrio y un 15\% refiere que es algo que afecta el estado de ánimo. Estas definiciones aunque son claras fueron correctas en épocas anteriores.

Por otro lado, un 55\% de la población define estilo de vida saludable como: las características o condiciones saludables propias de la vida personal; un 20\% lo conceptualiza como un rumbo, camino, régimen, acción, pensamiento y/o forma de vida; que vaya a favor de la salud. Estas respuestas emitidas se acercan al concepto real, donde se entiende el estilo de vida como:

"Las formas de conducirse tales como los hábitos o los comportamientos sostenidos, de los individuos y los grupos sociales y que son resultado de la interacción de factores de tipo material (vivienda, alimentación, vestido, servicios de saneamiento básico y tecnología), social (organización a la que se pertenece) e ideológico (ideas, valores y creencias), los cuales proporcionan las condiciones que determinan un estado de salud y la prolongación de la vida” (Villalobos, LB. 1989, p. 60).

Respecto al tema de la sexualidad el $25 \%$ de las personas entrevistadas la conceptualizaron como compartir y disfrutar con las otras personas y otro $25 \%$ aseguro que la sexualidad es la relación e interacción con la pareja. Sin embargo, la sexualidad es además, una forma de expresión del ser humano; el disfrute del amor, del placer y del goce son elementos enriquecedores en lo personal, familiar y social, lo cual contribuye a un estilo de vida saludable.

Un definición de sexualidad es la siguiente: 
“La sexualidad es más que la actividad física genital. La sexualidad comprende todo el ser. Incluye el sentido de feminidad o masculinidad, incluye también dimensiones del ser de cada persona. Además, la sexualidad esta influida por los valores, las conductas, las relaciones con otros y la necesidad de establecer un acercamiento emocional con los demás”

(MacLaren, 1995. s.p: Potter \& Perry 2003, p. 580).

Por otra parte, un 95\% afirmó sentirse satisfecho con su vida sexual. De acuerdo a lo anterior, Potter y Perry (2003) expresan que para los adultos la intimidad y la sexualidad son temas importantes tanto si tienen una relación sexual, han elegido abstenerse, estén solteros, sean homosexuales o sean viudos; cualesquiera que sean las circunstancias, la actividad sexual se define como una necesidad básica, pero el deseo sexual puede ser canalizado de forma sana en otras formas de intimidad.

Con respecto al estrés, el 30\% de la población afirma que los conflictos familiares son fuentes de estrés, mientras que para un $20 \%$ es la enfermedad, hospitalización y la muerte. Por otro lado, el $45 \%$ de los y las trabajadoras del Núcleo identificó los picos de trabajo como la principal fuente de estrés, un 30\% los estímulos auditivos altos y las relaciones interpersonales difíciles otro 30\%. Dentro de las situaciones económicas, el 40\% de la población en estudio afirmó que los ingresos insuficientes son una fuente de estrés. Igualmente, la relación de dependencia entre las variables padece de estrés y deudas financieras, demostró que existe una probabilidad asociada de 0,002 ; es decir, que es estadísticamente significativa al 5\%. Este resultado denotó que las personas que tienen deudas financieras tienen mayor probabilidad de padecer estrés. Además, al relacionar las variables tiempo de laborar y padece estrés se obtiene una probabilidad asociada de 0,009; lo que demuestra que es estadísticamente significativa al 5\%, y cuyo resultado denotó que 
entre más años se tenga de laborar para la institución aumenta la probabilidad de padecer estrés.

Como se observa en las estadísticas anteriores, en la vida diaria, existen diversas situaciones que pueden causar estrés dependiendo de la individualidad; ya que según Neal, C. (1995), el estrés aparece siempre que un hecho real o un peligro aparente perturbe nuestro equilibrio psicológico y/o fisiológico; hecho que puede ser una situación física o inclusive un peligro conceptual; ya que no hace falta que exista un peligro real o una amenaza presente para causar una reacción de estrés.

Entre las manifestaciones del estrés el 60\% de las y los participantes en el estudio manifiestan el estrés por medio de dolores musculares y el $40 \%$ por medio de cefaleas. En relación con las manifestaciones laborales, el 40\% afirman manifestarlo con una sensación de cansancio al realizar el trabajo, el $15 \%$ de la población al necesitar descansos intermitentes; además, requieren mayor nivel de atención para realizar el trabajo un 15\% y se duerme mientras realizan el trabajo otro 15\%. Según las manifestaciones psicológicas, el 55\% afirma manifestar estrés mediante una preocupación por las cosas, el 45\% se alteran, irritan o molestan fácilmente y el 40\% aumenta el consumo de alimentos.

Al respecto se afirma que: 


\begin{abstract}
“Cuando aparece el estrés, ya sea bajo buenas o malas circunstancias, las reacciones pueden ser física, emocional y de la conducta. El cuerpo responde al estrés con una reacción de lucha o escape. Las reacciones físicas involuntarias incluyen: la tensión muscular, la aceleración del ritmo cardiaco y un aumento en la adrenalina que trae como resultado un estado de alerta. Emocionalmente, quizás nos pondríamos irritables, deprimidos y cosas por el estilo. El estrés puede afectar el comportamiento creando distracciones que no nos permiten tener un funcionamiento adecuado. Es posible también que haya un cambio en la reacción hacia las personas que nos rodean, así como un aumento o disminución en nuestra actividad y otras cosas más" (Neal, C.1995, pp. 8-9).
\end{abstract}

Finalmente, en lo que respecta a las estrategias de afrontamiento del estrés que utilizan los y las trabajadoras del Núcleo, el 45\% conversan, un 45\% duerme, el 35\% realiza ejercicios y un 30\% realiza actividades recreativas. Al respecto, Potter y Perry afirman que, “...el ejercicio regular, el descanso adecuado y una dieta nutritiva son opciones de estilo de vida positiva que pueden reducir el estrés.” (Potter \& Perry 2003, p. 670). Además, Neal, C. (1995) propone como estrategias para reducir el estrés las siguientes: descansar lo suficiente, tomar tiempo para uno mismo, buscar apoyo emocional, recibir un masaje, reírse, expresar verbalmente los sentimientos, meditar, orar, leer, salir a pasear, entre otros.

\title{
CONCLUSIONES
}

$\checkmark$ Se evidenció que los y las trabajadoras del Núcleo se encuentran satisfechas con respecto al espacio físico, al orden, al aseo, la decoración y la infraestructura de la institución; así mismo refieren sentirse motivadas y satisfechas al iniciar su jornada laboral.

$\checkmark$ La mayoría de los y las participantes expresaron no estar conformes con respecto a la seguridad del ambiente laboral ni con la ventilación; además, consideran que existe ruido continuo y de alta intensidad. Así mismo, se identificó que no existe clasificación de desechos sólidos y medidas de seguridad (como salidas de emergencia y rotulación). 
$\checkmark$ La mayoría de las personas califican como "bueno” la iluminación, el aseo, el orden y la ventilación.

$\checkmark$ La evolución del término salud hacia un proceso ligado a la enfermedad y definido como una construcción social, trae como consecuencia que las poblaciones actuales deban integrarse de forma activa a la participación social, es decir, nuestra sociedad se encuentra adscrita a un quehacer colectivo, el cual se fundamenta en la práctica de estilos de vida saludable que a la vez es el promotor de una mejor calidad de vida.

$\checkmark$ A pesar de que el concepto de salud ha cambiado, en la actualidad muchos continúan trabajando bajo un enfoque predominantemente curativo en lugar de preventivo.

$\checkmark$ Las personas, en ocasiones no identifican cuáles son las actividades recreativas que estimulan la salud.

$\checkmark$ La mayoría de la población cuenta con un ingreso suficiente para satisfacer sus necesidades básicas y que no asumen por sí solas los gastos económicos del Núcleo familiar.

$\checkmark$ El 95\% presenta deudas financieras, aspecto que podría ser un factor de riesgo; ya que en este estudio se determinó que existe una relación significativa entre el hecho de tener deudas financieras y el padecer estrés.

$\checkmark \quad$ El total de las personas participantes en el estudio cuentan en sus comunidades con los servicios básicos de agua potable, recolección de desechos sólidos, seguridad, electricidad y zonas verdes, lo cual beneficia directamente la calidad de vida de cada uno de ellos y ellas.

$\checkmark$ Los y las trabajadoras tienen como antecedentes patológicos familiares de enfermedades crónicas; dichos antecedentes los y las predisponen a padecer en un futuro estas enfermedades dependiendo del estilo de vida que tengan.

$\checkmark$ Menos de la mitad de las personas del estudio no realizan actividades recreativas como parte de su rutina diaria; aspecto que se relaciona con el hecho de que nuestra sociedad está caracterizada por un acelerado ritmo de vida, donde las personas no se otorgan un espacio para descansar ni recrearse.

$\checkmark$ Casi la totalidad de los sujetos padece de estrés, cuya principal causa son aspectos relacionados con el trabajo.

$\checkmark$ Se encontró que un porcentaje significativo de esta población mantiene hábitos poco saludables como el consumo de bebidas alcohólicas y tabaco, los cuales se ven muchas veces influenciados por la sociedad. 
$\checkmark$ Se identificó que la concepción que los y las trabajadoras tienen sobre el proceso saludenfermedad repercute en la frecuencia y las razones por las cuales ellos y ellas visitan los centros de salud; ya que ninguno asiste para promocionar su salud y prevenir enfermedades.

\section{RECOMENDACIONES.}

$\checkmark$ Al crear e implementar programas orientados a la promoción de estilos de vida saludables, los y las enfermeras deben considerar las características del ambiente laboral en el cual se desenvuelven los y las participantes.

$\checkmark$ Las jefaturas o encargados y encargadas de salud ocupacional de las diferentes organizaciones laborales deben brindar en cada uno de los lugares de trabajo, un espacio físico para que los y las trabajadoras puedan realizar actividades ya sean de relajación, ejercicios y recreación; promoviendo así un estilo de vida saludable.

$\checkmark$ Es importante que las instituciones y empresas promuevan ambientes laborales con características que favorezcan la práctica de estilos de vida saludable, y que contribuyan con la calidad de vida de los y las trabajadoras.

$\checkmark \quad$ En todo momento en que se desee educar a una población adulta, es necesario que los y las enfermeras tomen en cuenta las necesidades educativas de la misma, así como las preferencias de esta para llevar a cabo el proceso de enseñanza-aprendizaje, de forma exitosa.

$\checkmark$ Al implementar, un programa para promover estilos de vida saludable, es necesario que los y las enfermeras, realicen un análisis amplio de dichos estilos, por medio de un instrumento como el historial de enfermería, el cual permite conocer de manera integral aspectos de la vida de las personas.

\section{BIBLIOGRAFÍA.}

Adam, Félix (1987). Andragogía. Caracas: INSTEA.

Aguilar Herrera, A. (1992) Normas de ventilación (Primera parte). Tesis de Licenciatura en Ingeniería Mecánica, Escuela de Ingeniería Mecánica, Facultad de Ingeniería, Universidad de Costa Rica.

Castro, L; Peralta, T; Quesada, C \& Sibaja, A. (1997). Elaboración de un recetario con preparaciones modificadas para la prevención de enfermedades crónicas. Seminario de graduación de Licenciatura en Nutrición, Escuela de Nutrición, Facultad de Medicina, Universidad de Costa Rica.

Gerin, S (1998). Dormir bien para vivir mejor. Barcelona: Ediciones Robin Book.

Instituto Nacional sobre Alcoholismo. Departamento de educación (1983). Alcohol y Alcoholismo: información genera. (Cuarta edición). San José: INSA.

Ivancevich, J \& Matteson, M (1989). Estrés y trabajo: una perspectiva gerencial. (2da edición). México D.F.: Editorial Trillas

López Jiménez, H (1993). El tabaquismo. San José: sin editorial. 
Martínez, R; Sánchez, R. (1999). El Concepto de Salud. Achio, M \& Carballo, R. (2003). Antología de lecturas: Curso Sociología de la Salud. (pp. 99-109). San José: Universidad de Costa Rica.

Martínez, R; Sánchez, R. (1999). Dimensión Económica de la Salud. Achio, M \& Carballo, R. (2003). Antología de lecturas: Curso Sociología de la Salud. (pp. 109-114). San José: Universidad de Costa Rica.

Martínez, R; Sánchez, R. (1999). Relación entre Economía y Salud. Achio, M. \& Carballo, R. (2003). Antología de lecturas: Curso Sociología de la Salud. (pp. 114-123). San José: Universidad de Costa Rica.

Melgosa, J. (1997). Nuevo estilo de vida isin estrés!. Madrid: editorial Safeliz.

Ministerio de Salud (2003). Política Nacional de Salud 2002-2006. San José: Ministerio de Salud.

Neal, C. (1995). 52 maneras de reducir el estrés en tu vida. Florida: Editorial Caribe, INC.

O’ Brien, G. (1998). El estrés laboral como factor determinante de la salud. En: Buendía, J. (Ed.), Estrés laboral y salud (pp. 61-77). Madrid: Editorial Biblioteca nueva.

Orem, D. (1983). Normas practicas en enfermería. Madrid: Ediciones Pirámide, SA

Organización Mundial de la Salud. (1990). Dieta, Nutrición y Prevención de Enfermedades Crónicas (OMS serie de informes técnicos No. 797). Madrid: OMS.

Organización Panamericana de la Salud. (2002). ¡Muévete América! Una vida activa te da salud y energía. Washington, DC: OPS/OMS.

Osorio Correa, E. (2000, Agosto). Ejes de reflexión para la búsqueda de un sentido a la recreación. Apuntes para el sexto Congreso Nacional de Recreación. Bogotá: Sin editorial.

Potter, P \& Perry, A. (2003). Fundamentos de Enfermería (5 ed). (Vol. 1). Barcelona: Harcourt/OCÉANO

Quirós R, E. (1994). Este cuerpo que no habitamos: Alternativas del manejo del estrés en las mujeres. San José: Comisiones mujer, salud y desarrollo para Centroamérica, OPS/OMS.

Selye, H (1956). The stress of Live. New York: McGrawHill.

Villalobos, LB. 1989. Salud y sociedad: Un enfoque para Centroamérica. San José: ICAP. 